\title{
Electrowetting Force and Velocity Dependence on Fluid Surface
}

\section{Energy}

Qi Ni

University of South Florida

Daniel E. Capecci

University of South Florida

Nathan B. Crane

Brigham Young University - Provo, nbcrane@byu.edu

Follow this and additional works at: https://scholarsarchive.byu.edu/facpub

Part of the Other Mechanical Engineering Commons

\section{Original Publication Citation}

Q. Ni, D. E. Capecci, and N. B. Crane, "Electrowetting Force and Velocity Dependence on Fluid Surface Energy," Micro and Nanofluidics, 2015, DOI: 10.1007/s10404-015-1563-7.

\section{BYU ScholarsArchive Citation}

$\mathrm{Ni}$, Qi; Capecci, Daniel E.; and Crane, Nathan B., "Electrowetting Force and Velocity Dependence on Fluid Surface Energy" (2015). Faculty Publications. 5345.

https://scholarsarchive.byu.edu/facpub/5345

This Peer-Reviewed Article is brought to you for free and open access by BYU ScholarsArchive. It has been accepted for inclusion in Faculty Publications by an authorized administrator of BYU ScholarsArchive. For more information, please contact ellen_amatangelo@byu.edu. 


\title{
Electrowetting Force and Velocity Dependence on Fluid Surface Energy
}

\author{
Qi Ni, Daniel E. Capecci, and Nathan B. Crane ${ }^{a)}$
}

Department of Mechanical Engineering, University of South Florida, 4202 E. Fowler Avenue, Tampa, Florida, 33620, USA

a) Author to whom correspondence should be addressed. Electronic mail: ncrane@usf.edu

\begin{abstract}
Electrowetting on Dielectric is a phenomenon in which the shape and apparent contact angle of a droplet changes when an electric field is applied across the droplet interface. If the field is asymmetric with respect to the droplet, then a net force can be applied to the droplet. In this work, we have measured the electrowetting force by confining the droplet shape beneath a glass plate and measuring the force on the plate. The force was measured as a function of voltage for a range of fluids with different surface energy. Measured forces show excellent agreement with predictions based on the Young-Lippmann equation with measured contact angles. Results also show that the electrowetting force is independent of fluid surface energy below saturation but that the peak force is proportional to the surface tension. This work shows that lowering the surface energy of the fluid can induce larger contact angle change under the same voltage, but it has no beneficial impact on the actuation force in droplet-based actuators. In contrast, velocity tests with deformable droplets show higher speeds for lower surface energy fluids, even above their saturation voltage. However, when the droplet's shape is restrained, the highest velocity is achieved with high surface energy fluids due to the larger electrowetting actuation forces applied.
\end{abstract}

Keywords: EWOD, electrowetting force, droplet, contact angle, droplet actuator, velocity, contact line friction. 


\section{Introduction}

Electrowetting on dielectric (EWOD) uses an external electric field to change the apparent contact angle of a droplet on a dielectric layer. For grounded droplet configuration, a conductive liquid and a metal substrate are separated by a thin dielectric. Voltage potential is applied between the droplet and the substrate. The contact angle of the droplet follows the Young-Lippmann equation until a certain point which is referred to as the contact angle saturation (Mugele and Baret 2005). EWOD is frequently employed in optical (Smith et al. 2006), display (Hayes and Feenstra 2003), and lab-on-chip technologies (Cho, Moon, and Chang-Jin Kim 2003) where fluid position and shape are the primary metrics, but it also shows promise in mechanical applications including pumps (Yoon 2001), grippers (Vasudev and Zhe 2008), switches and motors (Sen 2009; Takei, Matsumoto, and Shomoyama 2010).

For display and bio-sampling applications, motion velocity is a key metric as it improves video quality and through put, respectively. On the other hand, the maximum force the EWOD droplet can achieve is the primary interest in designing actuators, pumps, and grippers. However, only limited direct measurements of mesoscale electrowetting force have been reported previously. Common experimental approaches use either capillary rise experiments which calculate the electrowetting force from induced hydrostatic pressure difference (Chen and Hsieh 2006), or by relating the electrowetting force to the contact angle based on the Laplace pressure across the droplet/ambient interface (Berthier et al. 2007). Other techniques such as measuring the pressure change inside of a microfluidic channel (T. B. Jones et al. 2003), measuring the capacitance of the droplet (Verheijen and Prins 1999) or using an attached AFM tip (Guan et al. 2009) have also been explored. 't Mannetje et al. ('t Mannetje et al. 2013) has measured the force due to localized EW under applied AC signal, but these measurements are limited by the strength of the wetting to the small rod used to move the droplet. Due to the complex geometry and the dynamics of the contact line, direct force data is scarce.

This paper addresses this gap in experimental investigations in electrowetting forces and their dependence on the fluid properties. In this work, we address how the EW force varies with the applied electrical potential and the surface energy/ surface tension $(\gamma)$ of the droplet/ambient interface. First, the force relationships for electrowetting of a droplet with respect to the contact line length are developed. Experimental method for directly measuring the electrowetting force are described and the results are compared to the conventional contact angle approximation. Velocity of droplets of high and low surface energy fluids are compared between a free deformable drop and a constrained drop. Design considerations for EWOD actuators are discussed and the relationship of these measurements to prior velocity testing results are discussed. 


\section{Electrowetting Force}

For an arbitrary shape droplet during EWOD actuation, the change in contact angle is induced by the electric field between the fluid- dielectric interface. The force acting near the interface per unit length of contact line can be expressed as:

$$
\overline{\mathbf{F}}=\frac{\varepsilon_{0} * \varepsilon}{2 * \delta} * \overline{\mathbf{V}}^{2},
$$

where $\varepsilon_{0}$ is the permittivity of free space, $\varepsilon$ is the dielectric constant of the insulating layer, and $\delta$ the dielectric thickness (T.B. Jones 2009).The electrowetting force can be calculated by integrating around the droplet contact line covering the active electrode (see Figure 1):

$$
\mathrm{F}_{\text {Total }}=\int_{\mathrm{c}} \overline{\mathbf{F}} \cdot \widehat{\mathbf{n}} \mathrm{ds},
$$

where $\overline{\mathbf{F}}$ is the force vector and $\widehat{\mathbf{n}}$ is the unit normal to the direction of the force.

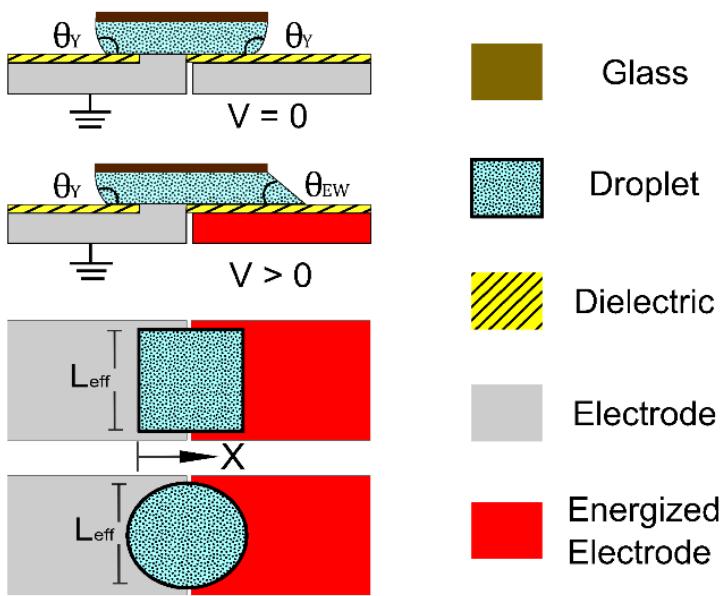

Figure 1: A square droplet is created by placing a glass plate over it. The droplet assumes the shape of the glass plate. One side of the droplet is grounded. When a voltage is applied to the other electrode, the contact line moves only slightly but a force is still applied to the glass plate.

We will consider the case of a droplet wetted to a glass plate placed on a hydrophobic $\left(\mathrm{CA}>90^{\circ}\right)$ substrate. For small droplet volumes, the droplet is well-approximated by a prism with the cross section of the glass plate (Crane, Mishra, and Volinsky 2010). For the simple cases of a rectangular plate or a circular plate that is halfway over the energized electrode (Figure 1), the total electrowetting force can be combined with the Young-Lippmann equation and simplified to: 


$$
\mathrm{F}=\frac{\varepsilon_{0} * \varepsilon}{2 * \delta} * \mathrm{~V}^{2} * \mathrm{~L}_{\mathrm{eff}}=\left(\operatorname{Cos}\left(\theta_{\mathrm{EW}}\right)-\operatorname{Cos}\left(\theta_{\mathrm{Y}}\right)\right) * \gamma * \mathrm{~L}_{\mathrm{eff}}
$$

where $\theta_{\mathrm{EW}}$ is the apparent contact angle under voltage, $\theta_{\mathrm{Y}}$ is the equilibrium contact angle when $\mathrm{V}=0$, $\gamma$ is the droplet/ambient interfacial tension, $\mathrm{L}_{\mathrm{eff}}$ is the length of the droplet normal to the force direction at the boundary between the two electrodes. Alternatively, the force can be found by calculating the derivative of the system energy with respect to a displacement in the direction of the force. For the case of a square droplet, the change in Leff with voltage and position is negligible so that the energy change in the system is the electrical energy stored in the capacitor formed between the droplet and the electrode (Crane, Mishra, and Volinsky 2010). The capacitive energy stored below a square droplet is (see Figure 1):

$$
\mathrm{E}=\frac{1}{2} * \mathrm{C} * \mathrm{~V}^{2}=\frac{1}{2} *\left(\frac{\varepsilon_{0} * \varepsilon * \mathrm{X} * \mathrm{~L}_{\mathrm{eff}}}{\delta}\right) * \mathrm{~V}^{2}
$$

where $(\mathrm{x} * \mathrm{~L})$ is the area of the droplet that's covering the working electrode. Taking:

$$
\mathrm{F}=\frac{\mathrm{dE}}{\mathrm{dx}}=\frac{1}{2}\left(\frac{\varepsilon_{0} * \varepsilon}{\delta}\right) * \mathrm{~V}^{2} * \mathrm{~L}_{\mathrm{eff}}
$$

Eq. 3 is recovered. It is important to note that according to Eq. 5, the force is independent of the interfacial tension of the fluids used. So below the contact angle saturation, the force should only be a function of the voltage applied.

\section{Experimental Setup}

\section{Electrowetting Force Measurement}

The experimental configuration is illustrated in Figure 2. A force sensor (NanoScience Instruments FT-270, resolution $2 \mu \mathrm{N}$ ) was bonded to a thin glass fiber (diameter $\sim 5 \mu \mathrm{m}$ ) using cyanoacrylate. On the other end of the fiber, a glass plate (13.5 $\mathrm{x} 4.5 \mathrm{x} 0.1 \mathrm{~mm})$ was attached using the same method. The glass plate was patterned with $1 \mu \mathrm{m}$ Cytop ${ }^{\circledR}$ to define two hydrophilic regions separated by a hydrophobic region. One droplet is placed in each hydrophilic regions. Due to the uneven pressure distribution inside the droplet under electrowetting actuation, the glass plate tends to tilt when a voltage was applied. Therefore, only the droplet away from the force sensor was actuated during force measurements; and the droplet which was closer to the sensor provided a counter moment to the plate to prevent rotation. As a result, the lateral force of electrowetting was translated through the droplet to the top glass plate and measured by the force sensor with negligible plate tilting. 


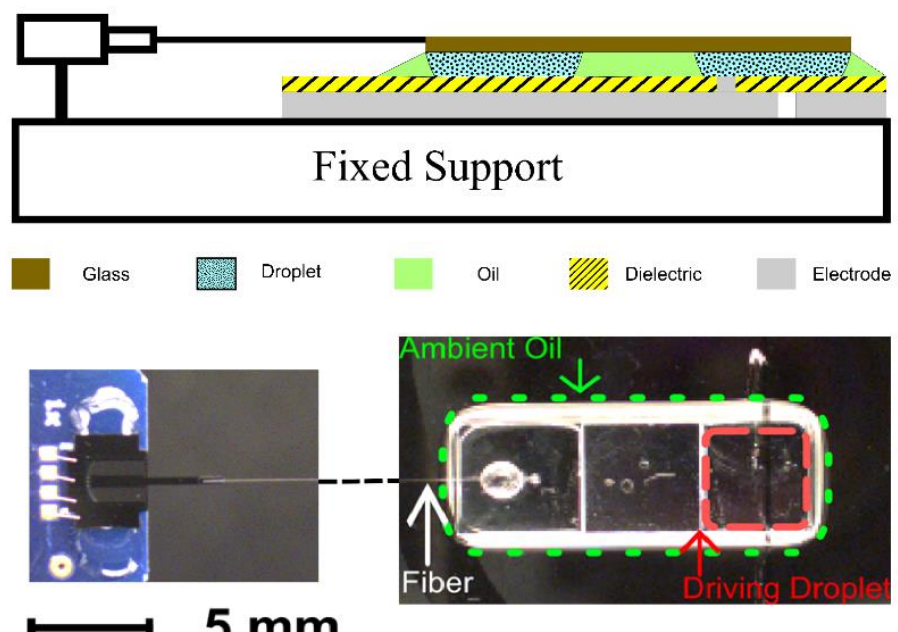

\section{$5 \mathrm{~mm}$}

Figure 2: Force measurement setup. The illustration on top is the measurement system viewed form the side. The lower left is a magnified view of the force sensor from top. The lower right is the top view of the glass plate with droplets and oil under the plate.

The substrate was fabricated by evaporating aluminum $(400 \mathrm{~nm})$ onto clean glass slides using electron-beam deposition. The aluminum was patterned to form two electrodes using photolithography. A $2 \mu \mathrm{m}( \pm 0.15 \mathrm{um}$, measured by Alpha-step profiolometer) Parylene C coating was deposited using a Parylene lab coater (Specialty Coating System, PSD2010). Finally, a $30 \mathrm{~nm}^{\text {Cytop }}{ }^{\circledR}$ top coat $^{2}$ was applied to render the substrate hydrophobic. Our group and others have reported anodic electrowetting with weak acid and aluminum improves electrowetting reliability (Khodayari, Carballo, and Crane 2012; Dhindsa et al. 2011). Therefore, the electrolyte base was chosen to be 0.1 M Citric Acid. Non-ionic, water soluble surfactant (Tergitol ${ }^{\mathrm{TM}}$ NP-8, CMC 61 ppm, DOW chemical company) was added with $0.001 \%$ and $0.005 \%$ by weight concentration. Ambient phase used were silicone oil (OS-30, Dow Corning) or $\mathrm{n}$ - hexadecane (99\% pure, Alfa Aesar).

The experimental procedures are listed as follows: (i) Before each test run, a scratch was made in the Parylene covering the grounding electrode to provide electrical ground to the droplet. (ii) The fluid of interest (a total of two droplets with volume of $10 \mu \mathrm{L}$ each) was deposited onto the two wetting regions on the top plate manually using a pipettor. (iii) The glass plate was lowered onto the substrate to allow the droplets to touch the surface. (iv) Ambient fluid $(10 \mu \mathrm{L})$ was pipetted to the gap between the glass plate and substrate. (v) A DC voltage ramp was applied at 1V/0.2 second and the reading from the force sensor was collected as well as the applied potential (see Figure 3). A total of 5 tests was performed and each test were done on a new location on the substrate. The total volume of the fluid used was fixed to be $30 \mu \mathrm{L}$ during all experiments and the gap height was maintained by the surface tension of the oil/air interface. Therefore, no significate height change was observed with different surfactant concentration. 


\section{To Force

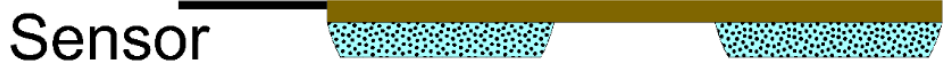

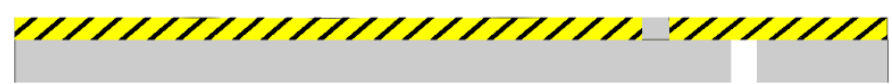
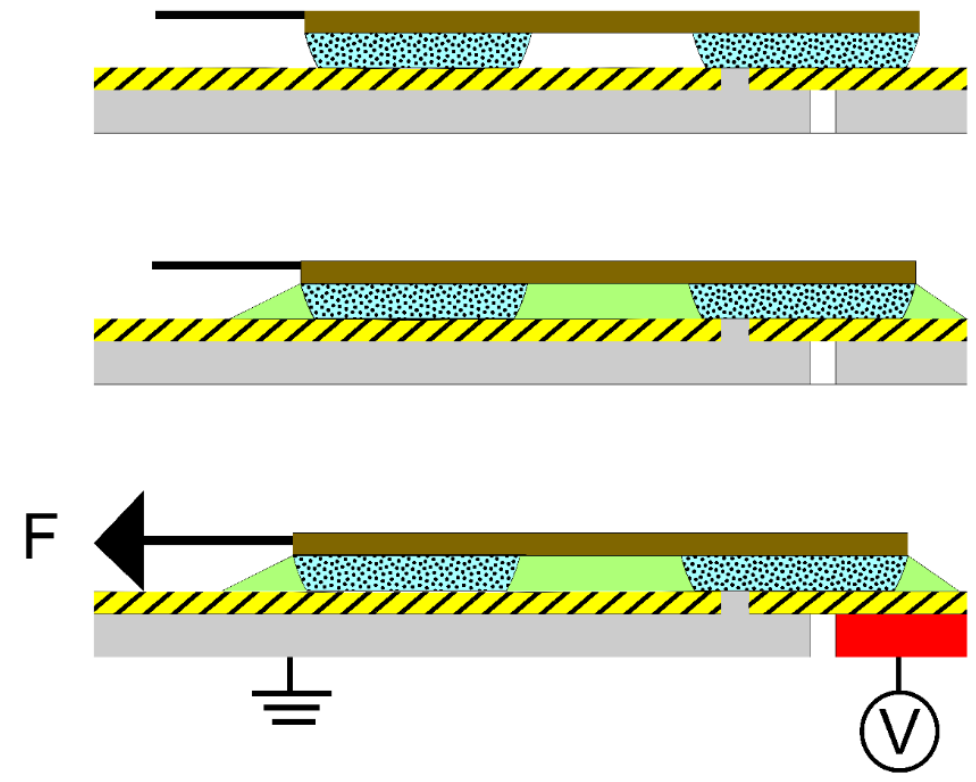

\section{Glass}

\author{
Droplet
}

\author{
Dielectric
}

\section{Electrode}

Oil

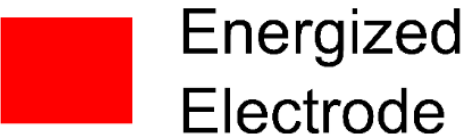

Figure 3: Experimental procedure. Droplets of testing fluids were deposited first onto the top plate. Then the plate was lowered to touch the electrowetting substrate. Ambient fluid was then added. When a voltage was applied, the force on the plate was measured and recorded.

\section{Contact angle measurements}

Contact angle measurement was done on a goniometer fitted with a digital camera and the images were processed using ImageJ software.

Contact angle was measured with the same voltage ramp as the force measurements. For solutions with surfactant, the voltage ramp was stopped at 80 Volts. For all other measurements, the voltage was ramped to 120 Volts. Again, a total of 5 tests was performed for each solution.

\section{Results and Discussion}

\section{Direct force measurements}


The measured actuation force is plotted vs. the applied voltage. As the figure shows, regardless of the concentration of the surfactant; the measured force followed the same slope until saturation. The peak force each fluid can achieve was proportional to the interfacial surface energy (see Figure 4).

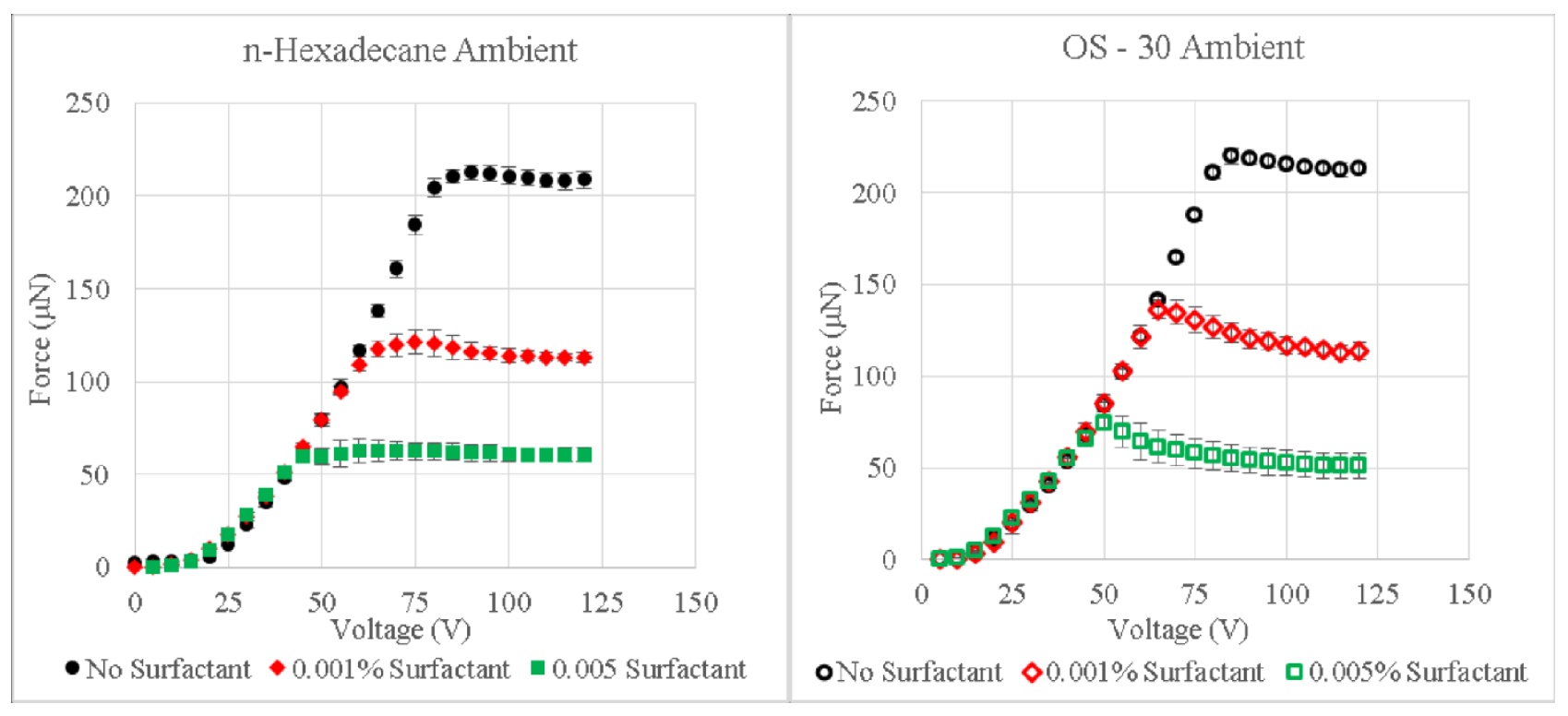

Figure 4: Measured electrowetting force vs. the applied voltage. The plot on the left shows the tests performed using n-Hexadecane as the ambient fluid and the plot on the right using OS-30 silicone oil. No surfactant: $0.1 \mathrm{M}$ citric acid. $0.001 \%$ surfactant and $0.005 \%$ surfactant: the percentage concentration by weight added to the base solution. The average values of 5 tests for each material combination are presented with the error bars showing the standard deviation of the results.

\section{Contact angle measurements}

The measured contact angle is plotted vs. voltage applied (see Figure 5). All combinations of droplet fluid and ambient fluid showed contact angle variation from $\sim 170^{\circ}$ to $\sim 60^{\circ}$. Surface tension $(\mathrm{mN} / \mathrm{m})$ was extracted from the contact angle measurements using methods described by Banpurkar et al. (Banpurkar, Nichols, and Mugele 2008) and the values are tabulated in Table 1. 


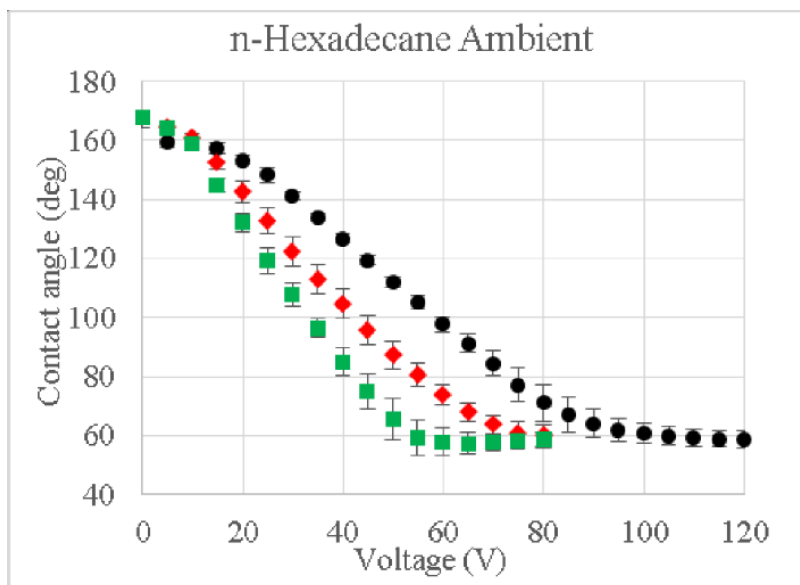

- No Surfactant $\bullet 0.001 \%$ Surfactant $=0.005 \%$ Surfactant

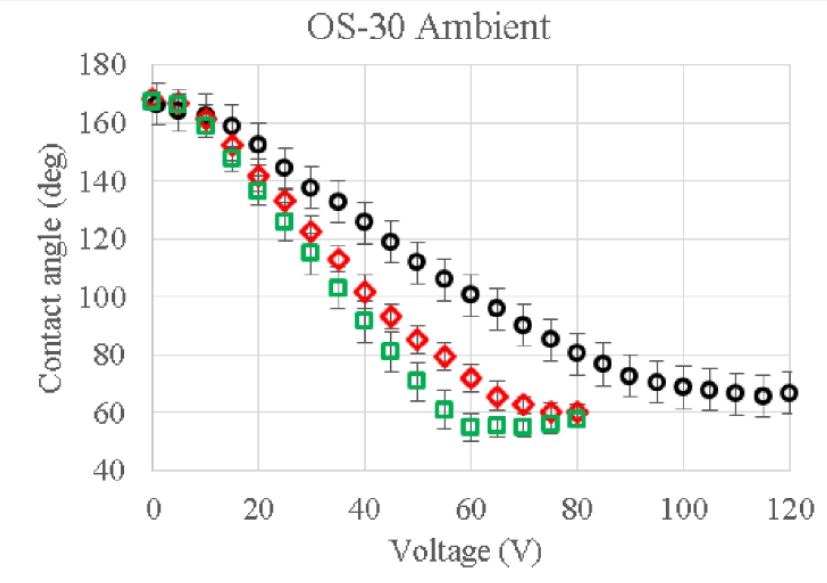

o No Surfactant $\diamond 0.001 \%$ Surfactant $\square 0.005 \%$ Surfactant

Figure 5: Measured contact angle vs. voltage applied. The figure on the left shows tests performed in n-hexadecane ambient and the figure on the right shows tests performed in OS-ambient. No surfactant: $0.1 \mathrm{M}$ citric acid. $0.001 \%$ surfactant and $0.005 \%$ surfactant: the percentage concentration by weight added to the base solution. The average of 5 tests is presented. Error bars show the standard deviation of the results.

Table 1 Surface tension values $(\mathrm{mN} / \mathrm{m})$ extracted from contact angle measurements. The rows are the actuation fluids, and the columns are the ambient oil. Five repetitions were performed for each test, and the average value and their standard deviation were listed.

\begin{tabular}{|c|c|c|}
\hline & $\mathrm{n}$ - Hexadecane ambient & OS -30 ambient \\
\hline 0.1 M Citric Acid & $30.9 \pm 2.3$ & $36.0 \pm 3.6$ \\
$0.001 \%$ by weight Tergitol added & $17.2 \pm 1.2$ & $18.9 \pm 1.1$ \\
$0.005 \%$ by weight Tergitol added & $10.7 \pm 0.7$ & $12.5 \pm 1.1$ \\
\hline
\end{tabular}

\section{Comparison between the measured force and the force calculated from contact angle}

In order to relate the measured force to the contact angle, the extracted interfacial tension and the average values of the measured contact angle, along with the plate width $\left(\mathrm{L}_{\mathrm{eff}}=4.5 \mathrm{~mm}\right)$ were used to calculate the electrowetting force using Eq. 3 . These results are compared to the measured electrowetting force in Figure 6. 


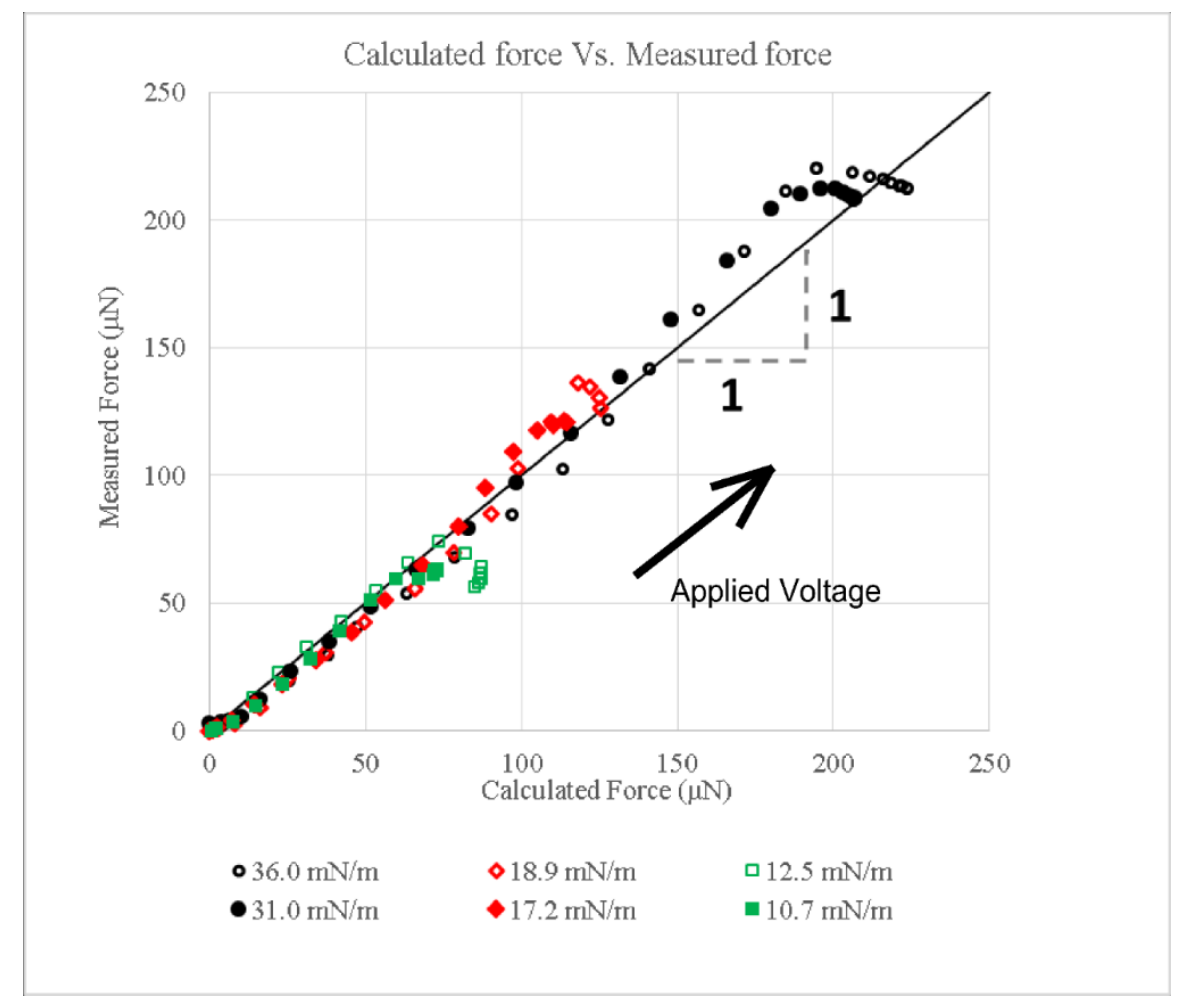

Figure 6: The average of measured electrowetting force is plotted against the average calculated force from contact angle. The relative surface tension values are used instead of the surfactant concentration. The straight line with the slope of 1 provides and comparison standard for those two methods used.

The measured electrowetting force agrees very well with the calculated force below the saturation voltage. The measured force for most fluids exceeded predictions as the voltage approached the saturation voltage before dropping below the prediction as the contact angle saturation voltage is exceeded. Although the cause of contact angle saturation is still under intense debate (Papathanasiou and Boudouvis 2005; Chevalliot, Kuiper, and Heikenfeld 2012), it is possible that in this case, geometry changes of the droplet could cause this increases due to a small increase in Leff prior to saturation.

The fact that the measured force dropped below the predicted force after contact angle saturation were somewhat difficult to interpret. It was suggested by Jones (T B Jones 2005) that the electrowetting force can still increase even after contact angle saturation. Jones also stated that there was no direct evidence to assume the contact angle saturation was limiting the electrowetting force. However, our measurements show only small excess of force near the contact angle saturation point after which the force drops below predictions. Thus, the contact angle saturation is a good prediction of the force saturation limits in these geometries. It is possible that these effects are due to size and geometric effects such as droplet spreading on the electrode and/or dewetting from the plate above the saturation voltage. Further work is necessary to address this force limit. 
To consider the data differently, the measured forces after the onset of saturation were first normalized by the effective length $(\mathrm{L}=4.5 \mathrm{~mm})$; then plotted against the electrowetting number $\left(\eta=\frac{1}{2}\left(\frac{\varepsilon_{0} * \varepsilon}{\gamma^{*}}\right) * \mathrm{~V}^{2}\right)$ to show the dependence of the force with surface tension of the fluids, then the same normalized forces were plotted against the surface tension times the electrowetting number $\left(\gamma * \eta=\frac{1}{2}\left(\frac{\varepsilon_{0} * \varepsilon}{\gamma * \delta}\right) *\right.$ $\mathrm{V}^{2}$ ) to show the voltage dependence of the electrowetting force, as shown in Figure 7. The figure highlights the design considerations for EWOD based devices.

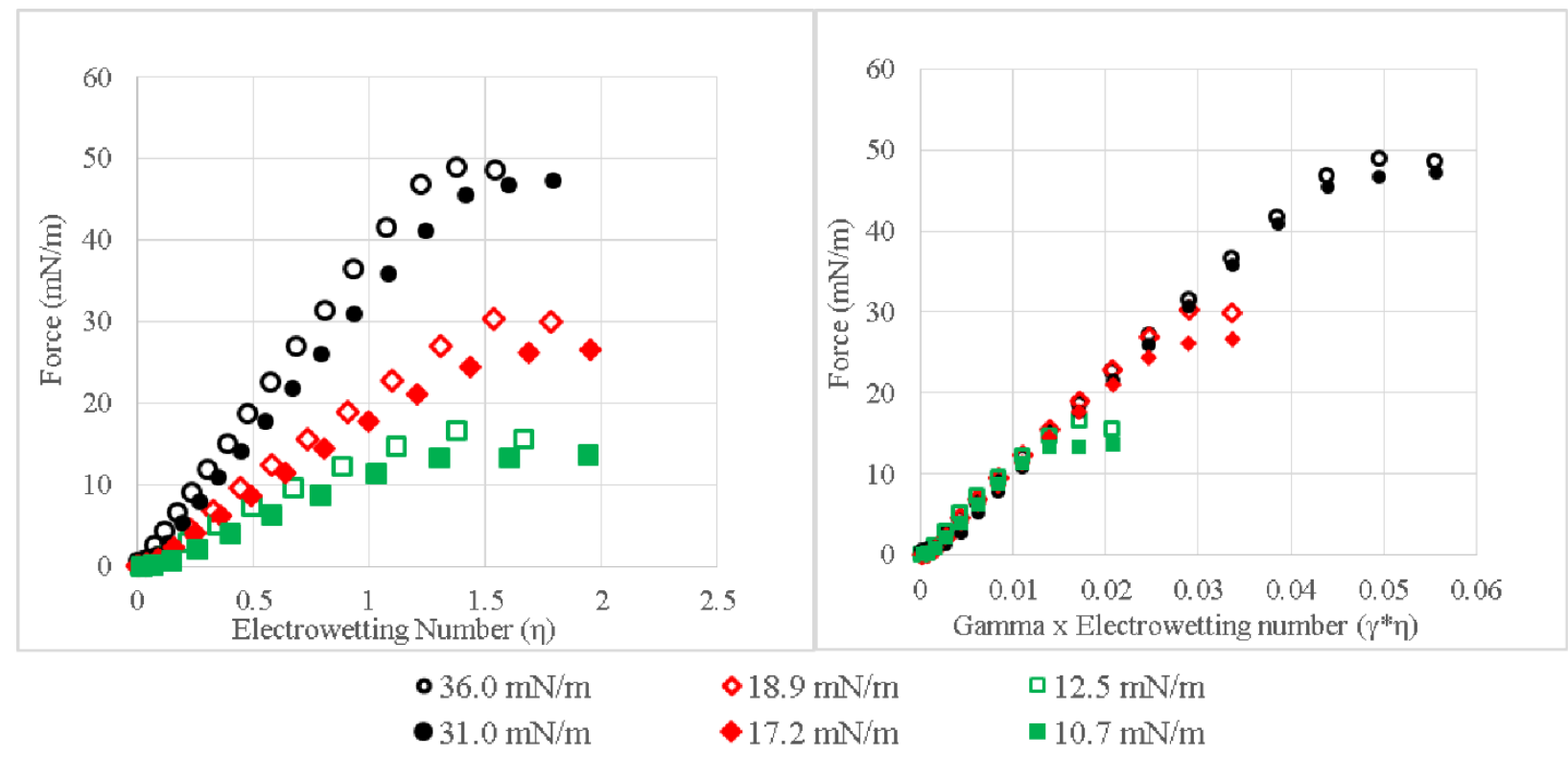

Figure 7: Measured unit force before saturation plotted against (a) electrowetting number ( $)$ ), left graph, (b)surface tension times electrowetting number $\left(\gamma^{*} \eta\right)$, right graph. Interfacial tension values are listed instead of material combination.

At the same electrowetting number, higher surface tension fluids provides higher actuation force (Figure 7a). While low energy fluids require less voltage to reach saturation, the actuation force is much larger with high surface energy fluids. Additionally, the electrowetting forces produced by all tested fluids follow the same linear relationship between force and $\mathrm{V}^{2}$ regardless of the interfacial tension of the fluid as predicted (Figure 7b). So the actuation force is only dependent on the voltage applied prior to saturation. Interestingly, the electrowetting force/length exceeded the interfacial energy of the fluid for all tested fluids, many by $50 \%$. Even with the uncertainty of the contact line length during the force experiments, the applied force/length has clearly exceeded the interfacial energy of the fluids. Thus, it is likely that the force was limited by either the stability of the droplet/substrate interface or the wetting force of the droplet to the glass plate on top. By decreasing the volume of the droplets and/or enhancing wetting to the top glass plate by adding roughness features to the plate, the electrowetting force might be increased further. 
In summary, for electrowetting based actuators, if lower voltage requirements is the primary design consideration, lower surface tension fluids should be used. If the maximum actuation force is the goal of the device, then higher surface tension fluids should be utilized.

\section{Considerations for Electrowetting Velocity}

As discussed above, actuation velocity is important to many electrowetting applications. Even where the force is the primary metric of concern, actuation velocity determines the response speed and actuator bandwidth. If the droplet were a rigid body, higher forces would be expected to produce higher accelerations and higher peak velocity. However, Lu et al. (Lu et al. 2007) has reported that the center of mass of the EWOD droplet reached higher velocity with higher electrowetting number which is contrary to this force based prediction.

To investigate the impact of electrowetting force on actuation speed, two additional sets of experiments were performed to compare velocities of different surface energy fluids in the traditional closed electrowetting configuration with a grounded top plate to the fixed shape droplet case studied here. Patterned substrates were fabricated using the same method described in earlier section. The size of the electrodes were $4 \times 4.5 \mathrm{~mm}$. For the first set of experiments, a cover plate coated with indium tin oxide (ITO) was used to provide electrical ground to the droplet. The ITO covered glass slides was also coated with $30 \mathrm{~nm}^{\text {Cytop }}{ }^{\circledR}$ to ensure the same hydrophobic surface property. The gap between the top plate and the substrate $(400 \mu \mathrm{m})$ was maintained by a plastic shim. The volume of the droplet was $10 \mu \mathrm{L}$ and the ambient fluid used was OS-30 oil. An initial voltage was applied to the electrode on the left at time $<0$ to maintain the position of the droplet at the beginning; then the voltage was switched to the electrode on the right at time $>0$. A digital camera above was triggered at $50 \mathrm{~Hz}$ to capture the evolution of the droplet as it moved towards the active electrode.

\section{Free deforming droplet}




\section{$\gamma=36.0 \mathrm{mN} / \mathrm{m}$

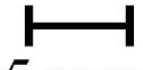 \\ $5 \mathrm{~mm}$}

$\gamma=18.9 \mathrm{mN} / \mathrm{m}$

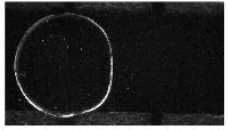

$\mathrm{t}<0$

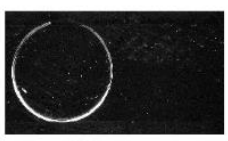

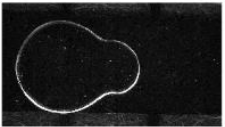

$\mathrm{t}=0.2$

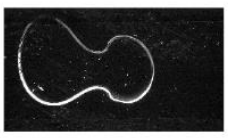

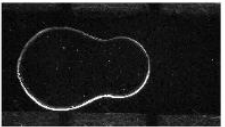

$\mathrm{t}=0.4$

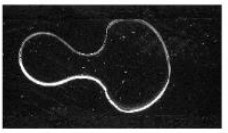

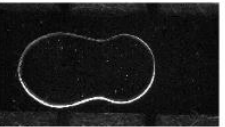

$\mathrm{t}=0.6$

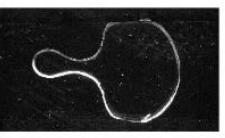

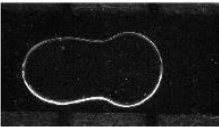

$\mathrm{t}=0.8$

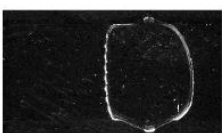

Figure 8: Comparing the motion of free deforming droplets sandwiched between two plates during electrowetting actuation. The top row is high surface tension fluid $(\gamma=36.0 \mathrm{mN} / \mathrm{m})$, and the bottom row is low surface tension fluid $(\gamma=18.9 \mathrm{mN} / \mathrm{m})$. The time which the image was taken is listed in seconds. The low surface tension fluid deformed much more during actuation and conformed to the electrode shape much easier. The scale bar is $5 \mathrm{~mm}$. Applied voltage: $70 \mathrm{~V}$, ambient fluid: OS-30. Droplet was grounded through an ITO covered top plate.

In our experiments, lower surface tension droplets reached the active electrode much faster than high surface tension droplet under the same applied voltage. This is true even when the applied voltage is well-above the saturation voltage of the low energy fluid. The images show that the low surface tension droplets were able to stretch more than the higher surface tension droplets at the same applied voltage (Figure 8). The characterization of the droplet deformation is beyond the scope of this paper, but contact line friction, viscosity, and contact angle hysteresis all contribute to this behavior (Shabani and Cho 2013; Song et al. 2008; Nelson and Kim 2012; Snoeijer and Andreotti 2013). Of all those factors, the dynamic contact angle hysteresis and the viscosity of both the ambient fluid and the droplet are thought to be the most relevant to the deformation of the droplets (see later section about dynamic contact angles and contact line friction). Under electrowetting actuation, the leading contact angle deforms under the applied potential and the contact line started to move. But the contact line at the top leading edge and the trailing edges (both top and bottom) was pinned due to contact angle hysteresis. The induced internal flow and the shear stress developed due to the viscosity contrast of the ambient and droplet causes the droplet to deform. The deformation on the leading edge of the droplet increases the effective contact line length on the active electrode to increase the force. Simultaneously, the low energy surface more readily deforms in response to the resistance of the ambient fluid to reduce the drag force on the droplet. Both effects would act to increase the droplet actuation velocity. The first effect would increase the force on the low energy droplet while the second would reduce the drag. Additionally, the contact line hysteresis or contact line friction, is often proportional to the surface energy. So the larger actuation force/contact line length may be partially offset by these contact line losses. These observations are consistent with the results of others (Lu et al. 2007; Ren et al. 2002) and additional studies are needed to provide a complete understanding. Given that lowering the surface tension can lower the operational voltage in the EWOD device, low surface tension fluids are favorable for the free droplets. 
If the higher speed of low surface energy droplets is related to their ability to change shape readily, this advantage should decrease or be reversed in the case of fixed shape droplets. As fixed geometries are able to exert the largest forces, this condition is important for maximizing actuation speed. In this case, high surface tension fluid would be expected to provide both high peak force and higher velocity. As a validation, velocity of fixed shape droplets of different fluid types was measured in the second set of experiments.

\section{Fixed shape droplet}

The same electrode design was used, but the top cover plate was replaced with a free circular glass plate (diameter: 8 mm, thickness: $100 \mu \mathrm{m})$ that was wet by the EWOD droplet. A scratch in the Parylene on one electrode was used to provide electrical ground. The oversized plate forced the droplet to wet the top plate and remain circular during motion. Also, the effective length of the droplet was constrained due to the fact that the plate diameter $(8 \mathrm{~mm})$ is much larger than the electrode width $(4.5 \mathrm{~mm})$. The experiments were performed in air with just $2 \mu \mathrm{L}$ silicon oil (OS-30) at the interface to minimize contact line friction. Although the experiments were done in air, the oil shell encapsulates the droplet/substrate interface so the electrowetting force should still depend on the droplet/oil surface tension values. The combined volume of the droplet and ambient oil resulted a gap height of $400 \mu \mathrm{m}$. Potential was applied to one of the electrodes and the movement of the droplet was captured at 50 frames/second. Afterwards, the images were analyzed using a

MATLAB routine to track the circular plate's position and the centroid of the plate was extracted for each frame. The displacement of the glass plate is plotted against the time in Figure 9.

At low voltage $(50 \mathrm{~V})$, the droplet displacement were similar for both high and low surface tension fluids. The low surface tension fluid moved slightly faster than the high surface tension fluid. It is possible that the same volume droplet would have a slightly larger contact area with lower surface tension. At high voltage $(70 \mathrm{~V})$, the velocity increased for both fluids. However, the constraining top plate limits the spreading and the higher surface energy fluid achieved much faster motion as would be expected from the EW force measurements. Thus, high surface energy fluids are preferred for electrowetting actuations when high velocity of a constrained droplet is needed or large actuation forces are desired. 


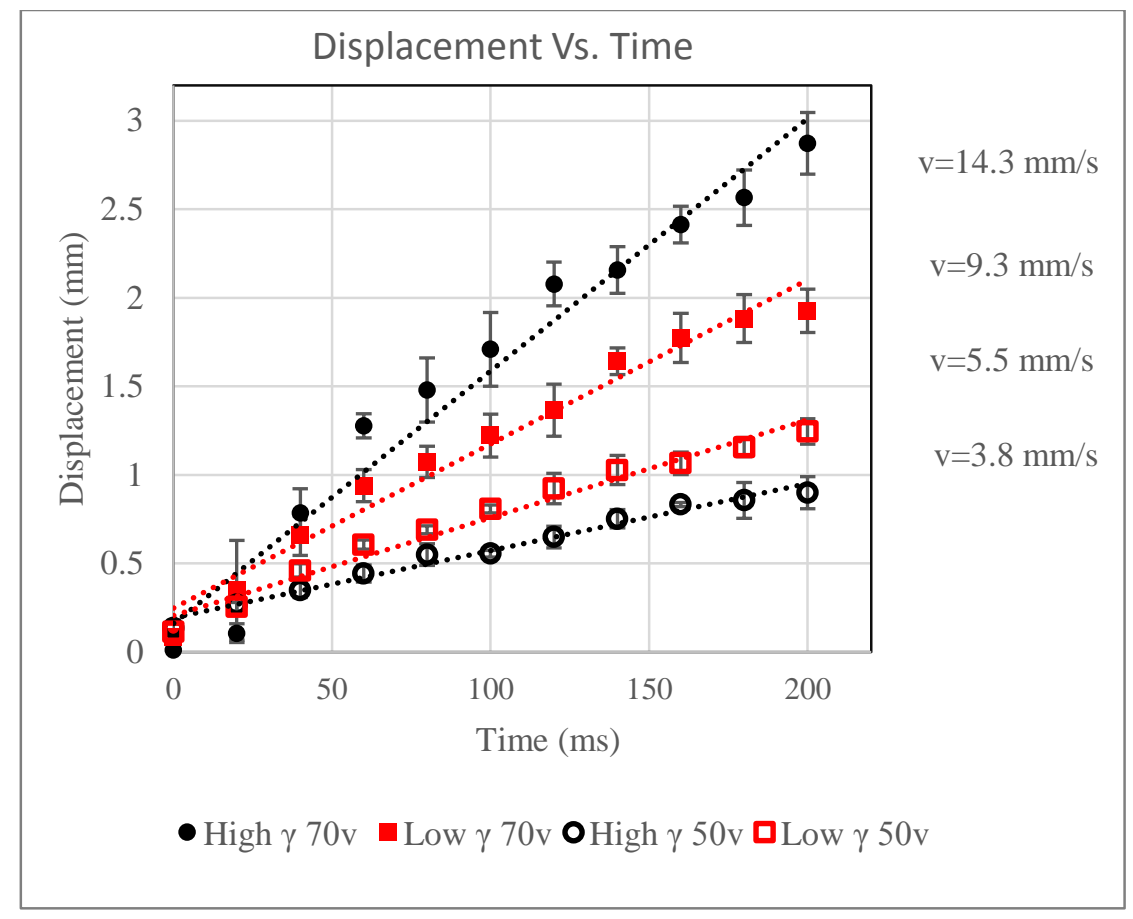

Figure 9: Plate speed under electrowetting actuation. A circular plate was carried by the EWOD droplet. The relative displacement of the plate $(\mathrm{mm})$ is plotted against the time $(\mathrm{ms})$ after the voltage was applied. High surface tension fluid was $0.1 \mathrm{M}$ citric acid (36.0 $\mathrm{mN} / \mathrm{m})$ and the low surface tension fluid $(18.9 \mathrm{mN} / \mathrm{m})$ was $0.001 \%$ surfactant by weight. The maximum voltage $(70 \mathrm{~V})$ was below both fluid's saturation voltage. The average velocity of the plate is listed on the right. The average value of 3 tests are presented and the error bars are the standard deviation of the data.

\section{Friction effect}

As discussed earlier, the forces oppose the motion is due to the combined effect of viscosity of the fluid, the surface tension and the contact angle variation during motion (dynamic contact angle). A large amount of detailed studies has been conducted for droplets with a moving contact line for both droplet spreading (Shikhmurzaev 1993; Terence D Blake 2006; Snoeijer and Andreotti 2013) and electrowetting forced wetting (T. D. Blake, Clarke, and Stattersfield 2000; Decamps and De Coninck 2000; Nelson, Sen, and Kim 2011).

The frictional force is usually thought to be proportional to the capillary number $\mathrm{Ca}\left(\mathrm{Ca}=\frac{\mu * \mathrm{U}}{\gamma}\right)$, where $\mu$ the viscosity and $\mathrm{U}$ the contact line speed.

For a fixed shape droplet (in our case, a thin circular cylinder shaped droplet), the friction variation can be measured by simply drag the droplet at fixed speed. Refer back to Figure 2, the same force measurement setup was used to drag the same fixed shape droplet across the same hydrophobic substrate. A linear stage (UTS100CC, Newport Corp.) was used to move the substrate at given velocity and the sensor was fixed to the support. The tests were performed for low and high surface tension fluids at $2.5,5,7.5$ and 15 mm/s. The initial inertial effect was discarded and only the forces at steady state velocities were reported. The measured forces were normalized 
by the plate diameter $(8 \mathrm{~mm})$ and then plotted against the velocity for each combination (Figure 10) and the insert shows the normalized force vs. the capillary number $\mathrm{Ca}$ (using $1 \mathrm{mPa} \cdot \mathrm{S}$ for $\mu$ ).

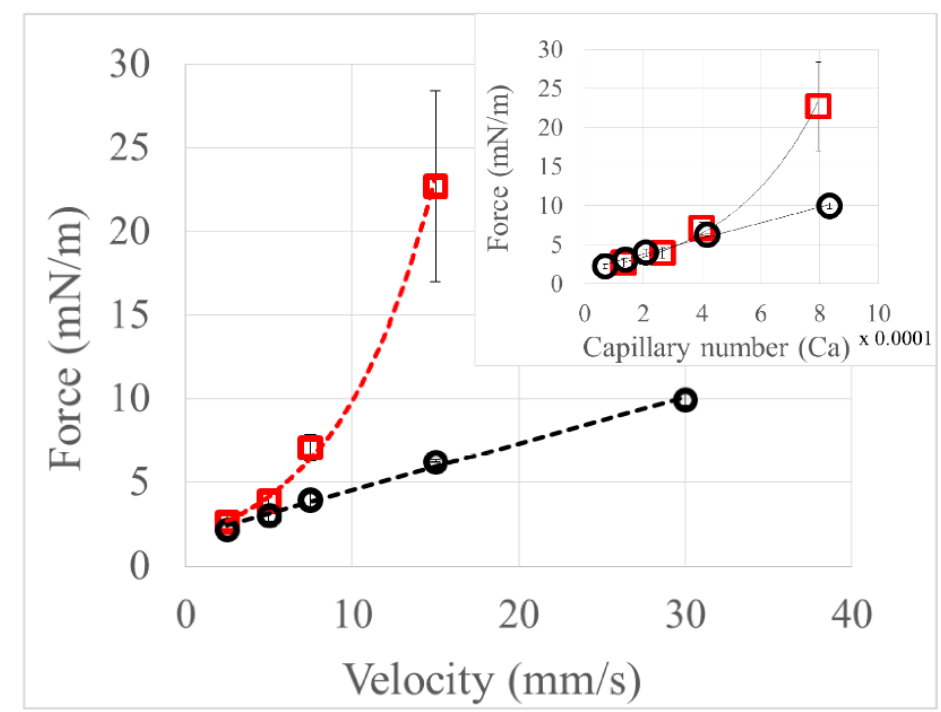

Figure 10: Normalized friction force Vs. velocity. The velocity for the tests were 2.5, 5, 7.5 and $15 \mathrm{~mm} / \mathrm{s}$ for all fluids with the additional $30 \mathrm{~mm} / \mathrm{s}$ for the high surface tension fluid only. The squares are the low surface tension fluids $(\gamma=18.9 \mathrm{mN} / \mathrm{m})$ and the circles are high surface tension fluid $(\gamma=36.0 \mathrm{mN} / \mathrm{m})$. Each data point represents the average of three runs and the standard deviation is presented. The insert is the same force plotted against the capillary number $(\mathrm{Ca})$.

During the experiments, a dynamic force oscillation was observed around $\mathrm{Ca} \sim 4 \times 10^{-4}$ but the force variation was low $(\sim 0.5 \mathrm{mN} / \mathrm{m})$. Plate tilting due to viscous shear was only observed for low surface tension fluid at $\mathrm{Ca} \sim 8 \times 10^{-4}$ (the last data point of low $\gamma$ ); also, both the drag force and the dynamic force oscillations started to increase after the average speed became stable for that particular case. The friction force for high surface tension fluid increased linearly with the capillary number. However, the friction force for low surface tension fluid had much larger gain when the capillary number increased to $\mathrm{Ca} \sim 8 \times 10^{-4}$. In the dynamic contact angle study conducted by Nelson et al. (Nelson, Sen, and Kim 2011), the dependency of contact line friction in terms of dynamic contact angle hysteresis without electrowetting was fairly weak at $\mathrm{Ca}<5 \times 10^{-4}$ and the contact angle hysteresis increased after $\mathrm{Ca}>1 \times 10^{-3}$ for water in air. Our experiments observed the same trend for low surface tension fluids where a large force increase was seen after Ca $>5 \mathrm{x} 10^{-4}$. It is unclear why higher surface tension fluids did not follow the same trend. It is possible that in our experiments the top plate was carried by the droplet and the weight of the glass plate contributed to the effect. It is also possible that surface tension changes the slope. Since the plate is not rigidly constrained, it could undergo some tilting that might impact the measured forces, but the plate appeared to remain parallel to the substrate during testing. 
Without provoking the hydrodynamic or molecular kinetic theory presented in earlier references (Shikhmurzaev 1993; Terence D Blake 2006; Snoeijer and Andreotti 2013); the data still provides some insight to the interpolation to earlier speed experiments. First, the friction force at given measured velocity (Figure 9) was extracted using fitted line from Figure 10. Assuming the measured friction forces represent the total force oppose the motion during electrowetting actuation, Table 2 summarizes the forces and their differences under actuation. The highly non-linear nature of the residual force (the difference between the applied force and the friction force) seems to indicate there are other additional dissipation mechanism which is related to both the surface tension and the velocity. Perhaps like it was pointed out in (Nelson, Sen, and Kim 2011), the stick slip behavior at high electrowetting number actuation has additional impact in the friction force and the electrowetting changed the localized flow condition around the contact line. Or it could be the assumption of measured friction force at steady state did not match the transient behavior of electrowetting actuation. More advanced analyzing methods is needed for future study.

Table 2: The measured electrowetting force and the friction force during actuation, all numbers are normalized by their respective length (mN/m)

\begin{tabular}{|c|c|c|c|}
\hline $\begin{array}{c}\text { Surface tension and measured } \\
\text { velocity }\end{array}$ & Electrowetting force & Friction force & Residual force \\
\hline $36.0 \mathrm{mN} / \mathrm{m}$ at $14.3 \mathrm{~mm} / \mathrm{s}$ & 36.5 & 5.7 & 30.8 \\
\hline $36.0 \mathrm{mN} / \mathrm{m}$ at $3.8 \mathrm{~mm} / \mathrm{s}$ & 18.7 & 8.7 & 15.9 \\
\hline $18.9 \mathrm{mN} / \mathrm{m}$ at $9.3 \mathrm{~mm} / \mathrm{s}$ & 30.0 & 4.5 & 14.4 \\
\hline $18.9 \mathrm{mN} / \mathrm{m}$ at $5.5 \mathrm{~mm} / \mathrm{s}$ & 19.0 & & \\
\hline
\end{tabular}

At low speed, both the low and high surface tension fluid were under the same actuation force. The contact line friction was slightly higher for the low surface tension droplet. However, the low surface tension droplet moved faster as seen in Figure 8 . When the contact line speed increased beyond $\mathrm{Ca} \sim 3 \times 10^{-4}$, the friction force increased as the speed of the contact line increased. The low surface tension droplet experienced much more friction force than the high surface tension droplet. The additional friction force limits the low surface tension droplet's speed at high voltage $(70 \mathrm{~V})$. For high surface tension fluid, the linear relationship with speed follows a less steep slope. So the high surface tension droplet can travel faster at high voltage. Since high surface tension fluids can reach higher velocity at given capillary number; they should be used for fixed shape droplet to maximize both the actuation force and speed. 


\section{Conclusion}

We have measured the electrowetting force by confining the geometry of the droplet. Below contact angle saturation, the measured force agrees well with the force predicted from contact angle measurements. The measured electrowetting force exceeds the predicted saturation point but declines with increased voltage beyond saturation. . Higher surface energy fluids achieve much larger forces than low surface energy fluids. However, droplet velocity is not directly related to the electrowetting force. Free droplet velocity is higher for lower surface energy fluids than higher surface energy fluids. It appears that droplet deformation has large impact in droplet transportation speed in EWOD of free droplets and the electrowetting number should be used to design EWOD devices for moving droplets as previously reported. However, for a droplet with fixed shape, the maximum velocity increases with higher surface tension fluids due to the limiting contact line friction force. So as a rule, for higher force/speed of EWOD actuators, higher surface tension fluids should be used.

\section{Acknowledgement}

The work is supported in part by the National Science Foundation thorough grant number CMMI-113075.

\section{References}

't Mannetje, Dieter, Arun Banpurkar, Helmer Koppelman, Michel H G Duits, Dirk van den Ende, and Frieder Mugele. 2013. “Electrically Tunable Wetting Defects Characterized by a Simple Capillary Force Sensor." Langmuir: The ACS Journal of Surfaces and Colloids 29 (31). American Chemical Society: 9944-49. doi:10.1021/la4015724.

Banpurkar, Arun G, Kevin P Nichols, and Frieder Mugele. 2008. “Electrowetting-Based Microdrop Tensiometer.” Langmuir : The ACS Journal of Surfaces and Colloids 24 (19). American Chemical Society: 10549-51. doi:10.1021/la801549p.

Berthier, Jean, Philippe Dubois, Philippe Clementz, Patricia Claustre, Christine Peponnet, and Yves Fouillet. 2007. “Actuation Potentials and Capillary Forces in Electrowetting Based Microsystems." Sensors and Actuators A: Physical 134 (2): 471-79. doi:10.1016/j.sna.2006.04.050.

Blake, T. D., A. Clarke, and E. H. Stattersfield. 2000. “An Investigation of Electrostatic Assist in Dynamic Wetting.” Langmuir 16 (6). American Chemical Society: 2928-35. doi:10.1021/la990973g. 
Blake, Terence D. 2006. "The Physics of Moving Wetting Lines.” Journal of Colloid and Interface Science 299 (1): 1-13. doi:10.1016/j.jcis.2006.03.051.

Chen, Jiann H, and Wen H Hsieh. 2006. "Electrowetting-Induced Capillary Flow in a Parallel-Plate Channel.” Journal of Colloid and Interface Science 296 (1): 276-83. doi:10.1016/j.jcis.2005.08.048.

Chevalliot, Stéphanie, Stein Kuiper, and Jason Heikenfeld. 2012. "Experimental Validation of the Invariance of Electrowetting Contact Angle Saturation.” Journal of Adhesion Science and Technology ahead-of-p (ahead-of-print). Taylor \& Francis: 1-22. doi:10.1163/156856111X599580.

Cho, Sung Kwon, Hyejin Moon, and Chang-Jin Kim. 2003. "Creating, Transporting, Cutting, and Merging Liquid Droplets by Electrowetting-Based Actuation for Digital Microfluidic Circuits.” Journal of Microelectromechanical Systems 12 (1): $70-80$. doi:10.1109/JMEMS.2002.807467.

Crane, Nathan B, Pradeep Mishra, and Alex A Volinsky. 2010. "Characterization of Electrowetting Processes through Force Measurements." The Review of Scientific Instruments 81 (4). AIP Publishing: 043902. doi:10.1063/1.3373945.

Decamps, C., and J. De Coninck. 2000. "Dynamics of Spontaneous Spreading under Electrowetting Conditions.” Langmuir 16 (26). American Chemical Society: 10150-53. doi:10.1021/la000590e.

Dhindsa, Manjeet, Jason Heikenfeld, Wim Weekamp, and Stein Kuiper. 2011. "Electrowetting without Electrolysis on Self-Healing Dielectrics." Langmuir: The ACS Journal of Surfaces and Colloids 27 (9). American Chemical Society: 5665-70. doi:10.1021/la1051468.

Guan, Li, Guicun Qi, Sheng Liu, Hui Zhang, Zhong Zhang, Yanlian Yang, and Chen Wang. 2009. "Nanoscale Electrowetting Effects Studied by Atomic Force Microscopy.” The Journal of Physical Chemistry C 113 (2). American Chemical Society: $661-65$. doi:10.1021/jp806538r.

Hayes, Robert A, and B J Feenstra. 2003. "Video-Speed Electronic Paper Based on Electrowetting." Nature 425 (6956): $383-85$. doi:10.1038/nature01988. 
Jones, T B. 2005. “An Electromechanical Interpretation of Electrowetting.” Journal of Micromechanics and Microengineering 15 (6). IOP Publishing: 1184-87. doi:10.1088/0960-1317/15/6/008.

Jones, T. B., Jesse David Fowler, Young Soo Chang, and Chang-Jin Kim. 2003. "Frequency-Based Relationship of Electrowetting and Dielectrophoretic Liquid Microactuation.” Langmuir 19 (18). American Chemical Society: 7646-51. doi:10.1021/la0347511.

Jones, T.B. 2009. "More about the Electromechanics of Electrowetting." Mechanics Research Communications 36 (1): 2-9. doi:10.1016/j.mechrescom.2008.08.012.

Khodayari, M., J. Carballo, and Nathan B. Crane. 2012. "A Material System for Reliable Low Voltage Anodic Electrowetting." Materials Letters 69 (February): 96-99. doi:10.1016/j.matlet.2011.11.060.

Lu, H.-W., K. Glasner, A. L. BertozzI, and C.-J. Kim. 2007. “A Diffuse-Interface Model for Electrowetting Drops in a Hele-Shaw Cell.” Journal of Fluid Mechanics $590 \quad$ (November). Cambridge University Press: $411-35$. http://journals.cambridge.org/abstract_S0022112007008154.

Mugele, Frieder, and Jean-Christophe Baret. 2005. "Electrowetting: From Basics to Applications.” Journal of Physics: Condensed Matter 17 (28): R705-74. doi:10.1088/0953-8984/17/28/R01.

Nelson, Wyatt C, and Chang-Jin “CJ” Kim. 2012. "Droplet Actuation by Electrowetting-on-Dielectric (EWOD): A Review.” Journal of Adhesion Science and Technology 26 (12-17). Taylor \& Francis: 1747-71. doi:10.1163/156856111X599562.

Nelson, Wyatt C, Prosenjit Sen, and Chang-Jin C J Kim. 2011. "Dynamic Contact Angles and Hysteresis under Electrowetting-onDielectric." Langmuir: The ACS Journal of Surfaces and Colloids 27 (16). American Chemical Society: $10319-26$. doi:10.1021/la2018083.

Papathanasiou, A. G., and A. G. Boudouvis. 2005. "Manifestation of the Connection between Dielectric Breakdown Strength and Contact Angle Saturation in Electrowetting.” Applied Physics Letters 86 (16). AIP Publishing: 164102. doi:10.1063/1.1905809.

Ren, Hong, Richard B. Fair, Michael G. Pollack, and Edward J. Shaughnessy. 2002. "Dynamics of Electro-Wetting Droplet Transport." Sensors and Actuators B: Chemical 87 (1): 201-6. doi:10.1016/S0925-4005(02)00223-X. 
Sen, P. 2009. "A Fast Liquid-Metal Droplet Microswitch Using EWOD-Driven Contact-Line Sliding." Journal of Microelectromechanical Systems 18 (1): 174-85. doi:10.1109/JMEMS.2008.2008624.

Shabani, Roxana, and Hyoung J. Cho. 2013. "Flow Rate Analysis of an EWOD-Based Device: How Important Are Wetting-Line Pinning and Velocity Effects?” Microfluidics and Nanofluidics 15 (5): 587-97. doi:10.1007/s10404-013-1184-y.

Shikhmurzaev, Y.D. 1993. "The Moving Contact Line on a Smooth Solid Surface.” International Journal of Multiphase Flow 19 (4): 589-610. doi:10.1016/0301-9322(93)90090-H.

Smith, Niel R., Don C. Abeysinghe, Joseph W. Haus, and Jason Heikenfeld. 2006. “Agile Wide-Angle Beam Steering with Electrowetting Microprisms.” Optics Express 14 (14). Optical Society of America: 6557. doi:10.1364/OE.14.006557.

Snoeijer, Jacco H., and Bruno Andreotti. 2013. "Moving Contact Lines: Scales, Regimes, and Dynamical Transitions.” Annual Review of Fluid Mechanics 45 (1). Annual Reviews: 269-92. doi:10.1146/annurev-fluid-011212-140734.

Song, J. H., R. Evans, Y.-Y. Lin, B.-N. Hsu, and R. B. Fair. 2008. “A Scaling Model for Electrowetting-on-Dielectric Microfluidic Actuators." Microfluidics and Nanofluidics 7 (1): 75-89. doi:10.1007/s10404-008-0360-y.

Takei, Atsushi, Kiyoshi Matsumoto, and Isao Shomoyama. 2010. “Capillary Motor Driven by Electrowetting.” Lab on a Chip 10 (14). The Royal Society of Chemistry: 1781-86. doi:10.1039/c001211d.

Vasudev, Abhay, and Jiang Zhe. 2008. “A Capillary Microgripper Based on Electrowetting.” Applied Physics Letters 93 (10): 103503. doi:10.1063/1.2978402.

Verheijen, H. J. J., and M. W. J. Prins. 1999. "Contact Angles and Wetting Velocity Measured Electrically.” Review of Scientific Instruments 70 (9). AIP Publishing: 3668. doi:10.1063/1.1149976.

Yoon, E. 2001. “A Micropump Driven by Continuous Electrowetting Actuation for Low Voltage and Low Power Operations.” In Technical Digest. MEMS 2001. 14th IEEE International Conference on Micro Electro Mechanical Systems (Cat. No.01CH37090), 487-90. IEEE. doi:10.1109/MEMSYS.2001.906585. 